\title{
Relative Peripheral Blood Volume Changes Induced by Premature Ectopic Beats and Their Role in Hemodialysis (Technical Note)
}

\author{
Eglè Grigonytė ${ }^{\mathrm{a}}$, Eduardo Gil ${ }^{\mathrm{b}}$, Pablo Laguna ${ }^{\mathrm{b}}$, Leif Sörnmo (corresponding \\ author $)^{\mathrm{a}}$ \\ ${ }^{a}$ Department of Biomedical Engineering and Center for Integrative Electrocardiology, Lund \\ University, Box 118, SE-221 00, Lund, Sweden. Email addresses: e.grigonyte@gmail.com, \\ leif.sornmo@bme.lth.se \\ ${ }^{b}$ CIBER de Bioingeniería, Biomateriales y Nanomedicina (CIBER-BBN), Spain, and Biomedical \\ Signal Interpretation \& Computational Simulation (BSICoS) at the Aragón Institute of \\ Engineering Research (I3A), University of Zaragoza, Spain. Email addresses: \\ edugilh@unizar.es,laguna@unizar.es
}

\begin{abstract}
Hemodialysis patients often suffer from cardiovascular disorders and uremic neuropathy, increasing the propensity to homeostatic imbalance that, in turn, may result in intradialytic complications like cramp, nausea, and, worse, hypotension. Ectopic beats, being abundant in such patients, may lead to imbalance through repeated, sudden drops in blood pressure. By exploring the properties of postectopic peripheral circulation recovery, treatment sessions prone to intradialytic complications may be better identified. This paper introduces a novel method for quantifying changes in peripheral blood volume due to ventricular or supraventricular premature beats (VPBs or SVPBs). Using the fingertip photoplethysmographic pulse waveform, VPB and SVPB-induced changes in relative peripheral blood volume are quantified by the postectopic pulse amplitude. Two parameters are proposed for characterizing (i) the initial drop in peripheral blood volume following an ectopic beat, and (ii) the degree of postectopic peripheral circulation recovery. A small set of data from 16 hemodialysis sessions in 9 hypotensionprone patients are used to illustrate the method. In asymptomatic sessions, the first parameter was found to be $8 \pm 13 \%$ (mean \pm std), whereas, in symptomatic sessions, it increased to $32 \pm 13 \%$, suggesting that postectopic pulse amplitude recovery is related to intradialytic complications; similar results were obtained for the second parameter. Postectopic pulse amplitude recovery may also be of
\end{abstract}


interest in other applications where relative changes in peripheral blood volume play a role.

Keywords: Hemodialysis, photoplethysmography, ectopic beats, intradialytic hypotension, postectopic circulation recovery.

\section{Highlights}

- A novel method for quantifying changes in peripheral blood volume due to ventricular or supraventricular premature beats.

- Two parameters are defined for characterizing postectopic pulse amplitude in the photoplethysmographic pulse waveform.

- Postectopic pulse amplitude recovery may be related to complications to complications during hemodialysis treatment. 


\section{Introduction}

Patients with chronic kidney disease undergoing dialysis are at high risk for cardiovascular disorders, since kidney failure affects cardiac structure and function through hemodynamic and metabolic changes [1], [2]. Resulting adverse cardiomyopathic and vasculopathic conditions are associated with sudden cardiac death, conduction abnormalities, and certain types of arrhythmias. In a recent study, ventricular arrhythmia was found to be present in $90 \%$ and supraventricular arrhythmia in $80 \%$ of patients with chronic kidney disease; both types of arrhythmia were exacerbated by dialysis [3]. Dialysis-related factors such as decreased circulating blood volume, sympathetic overactivity, and variation in metabolite concentrations were pointed out as causes to electrical instability of the heart.

Ventricular premature beats (VPBs) are frequent in hemodialysis patients [4], [5], and increase in number as the treatment session progresses and excess potassium is removed [6]. Furthermore, patients with regional wall motion abnormalities, ischemic heart disease, and left ventricular hypertrophy have more frequent VPBs during hemodialysis than patients without these conditions. Since VPBs are abundant in hemodialysis patients, interest has recently been directed to methods for exploring different aspects related to the occurrence of VPBs. For example, ECG-based analysis of heart rate turbulence (HRT) [7], has not only been established as a powerful predictor of mortality after myocardial infarction, but HRT analysis has been considered for intradialytic risk stratification. Using the standard HRT parameter "turbulence slope", propensity to acute intradialytic hypotension may be assessed [8], [9], see also [10].

Since it is inconvenient to wear ECG electrodes throughout a hemodialysis session, and calls for clinical staff who need to spend time on attaching electrodes, the much simpler photoplethysmographic (PPG) technique can be used since the accuracy of HRT parameters is not compromised when using the PPG [11]. The PPG is a noninvasive, optical technique suitable for tracking changes in microvascular blood volume; the sensor is usually attached to the fingertip [12]. In hemodialysis, the benefits of the PPG have been demonstrated for continuous intradialytic assessment of blood pressure [13], peripheral vascular regulatory function [14], hemodynamic stability [15], respiratory function [16], and fluid removal [17]. The prediction of acute intradialytic hypotension represents yet another problem addressed by using the PPG [18]: the method is based on the assumption that the global PPG amplitude, determined in a sliding minute-long window, provides a measure of change in relative blood volume in the microvascular tissue. 
This technical note introduces a novel method for quantifying changes in peripheral blood volume due to VPBs or supraventricular premature beats (SVPBs), information which may be used to identify patients at risk for intradialytic complications. The proposed method requires the availability of several ectopic beats, of ventricular and/or supraventricular origin, so that the PPG pulse amplitude can be computed in a short interval centered around the premature ectopic beats. It is hypothesized that changes in blood supply to the periphery due to the premature ectopic beats are reflected by changes in the pulse amplitude during the postectopic pulse interval. Here, such amplitude changes are referred to as pulse amplitude recovery (PAR). Two simple PAR parameters are proposed in Sec. 3, reflecting the initial circulatory disturbance and the degree of postectopic recovery of reduced peripheral microvascular circulation; both parameters are expressed as percentages. Using data acquired from hemodialysis patients (Sec. 2), the parameters are studied in Sec. 4 with respect to differences between sessions with and without complications.

It should be emphasized that HRT analysis resembles PAR analysis since both characterize the aftermath of ectopic beats. However, completely different physiological phenomena are characterized: while HRT relates to a short-term change in heart rate, PAR relates to a short-term change in peripheral blood perfusion.

\section{Materials}

The dataset consists of 11 hypotension-prone patients ( 7 females, age $64 \pm$ 12 years (mean \pm std)) with end-stage renal disease who underwent regular hemodialysis treatment thrice a week at Rigshospitalet, Copenhagen, Denmark. The patients were classified by a nephrologist as hypotension-prone from clinical history, such as the number of hypotensive events per month. Data acquisition was performed throughout the entire treatment session, lasting from 3 to $5 \mathrm{~h}$; a total of 28 sessions were acquired. A session was labeled symptomatic when an intradialytic complication occurred, i.e., hypotension, cramp, nausea or dizziness, and was accompanied by attention from the staff. The study was approved by the local ethics committee. The dataset has previously been studied in [18].

The machines Gambro AK 200 or AK 200 S (Gambro Lundia AB, Sweden) were used for patients who underwent hemodialysis and hemodiafiltration, respectively. The dialyzer filter was selected according to each patient's individual requirements. Data were acquired using external sensors operating in parallel with

the hemodialysis equipment. The electrocardiogram (ECG) was recorded with the Biopac ECG100C amplifier, and sampled at a rate of $1000 \mathrm{~Hz}$ with the Biopac 
MP150 data acquisition system (BIOPAC Systems, Inc., USA). The PPG signal was continuously acquired at the fingertip with a pulse oximeter (LifeSense(C), Medair AB, Sweden), also sampled at $1000 \mathrm{~Hz}$ using the Biopac MP150. The acquisition of ECG and PPG signals was time-synchronized since the same acquisition board was used. Thanks to this, matching of QRS complexes with the corresponding PPG waves is straightforward.

Based on the ECG, premature ectopic beats were manually annotated by two independent experts and grouped into VPBs and SVPBs. A premature ectopic beat was excluded from further analysis whenever another ectopic beat occurred within an "isolation interval" defined by $D_{1}$ beats before and $D_{2}$ beats after the premature ectopic beat. Moreover, if less than $K$ premature ectopic beats remained after application of the isolation criterion, the entire session was excluded from further analysis. In the PPG signal, segments for which the heart rate could not be reliably determined were excluded from further analysis [9]. The following parameter values were used in the present study: $D_{1}=6, D_{2}=16$, and $K=8$.

Applying these exclusion criteria, the original 28 sessions from 11 patients reduced to 16 sessions from 9 patients. For these 9 patients, VPBs were more frequent than SVPBs in symptomatic sessions (96\% vs. 53\%), whereas the reverse observation applied to asymptomatic sessions, i.e., SVPBs were more frequent than VPBs (47\% vs. 4\%), see Table 1.

In the PPG signal, different types of pulse pattern occur in response to a premature ectopic beat. Depending on the degree of blood pumping efficiency, an ectopic beat may or may not be associated with a distinguishable pulse [19]. Three types of PPG responses may be defined, namely, a pulseless response (denoted $P_{0}$ ), a pulse-related response (denoted $P_{1}$ ), and an indeterminate type of pulse response defined by an amplitude less than $10 \%$ of a normal PPG pulse amplitude, thus representing a transitional state between $P_{0}$ and $P_{1}$, see Fig. 1. The indeterminate type was not investigated in the present study.

\section{Methods}

\subsection{Signal preprocessing}

For the purpose of reducing the impact of high-frequency noise, the PPG signal was preprocessed with a lowpass FIR filter (cut-off frequency at $35 \mathrm{~Hz}$ ). In the filtered signal, the onset and peak locations of each pulse were determined using a derivative-based detector which finds the zeros of the first derivative followed by the determination of local minima and maxima [20]. 
Table 1: Patient information, presence of intradialytic complications during hemodialysis treatment, and the number of premature ectopic beats after application of exclusion criteria.

\begin{tabular}{cccccc}
\hline Patient: & Age/ & Compli- & \multicolumn{3}{c}{ Premature ectopic beats } \\
\cline { 5 - 6 } Session\# & Gender & cations & Quantity & VPBs, \% & SVPBs, \% \\
\hline $1: 1$ & $61 / \mathrm{F}$ & no & 41 & 46 & 54 \\
$1: 2$ & & no & 51 & 2 & 98 \\
$1: 3$ & & no & 9 & 11 & 89 \\
\hline $2: 1$ & $76 / \mathrm{M}$ & no & 44 & 82 & 18 \\
\hline $3: 1$ & $66 / \mathrm{M}$ & no & 494 & 88 & 12 \\
$3: 2$ & & yes & 448 & 100 & 0 \\
\hline $4: 1$ & $63 / \mathrm{F}$ & no & 15 & 7 & 93 \\
\hline $5: 1$ & $58 / \mathrm{F}$ & yes & 573 & 98 & 2 \\
$5: 2$ & & yes & 469 & 98 & 2 \\
$5: 3$ & & yes & 594 & 99 & 1 \\
$5: 4$ & & yes & 652 & 91 & 9 \\
\hline $6: 1$ & $56 / \mathrm{M}$ & no & 39 & 100 & 0 \\
\hline $7: 1$ & $59 / \mathrm{M}$ & no & 169 & 99 & 1 \\
\hline $7: 2$ & & yes & 80 & 93 & 7 \\
\hline $8: 1$ & $75 / \mathrm{F}$ & yes & 35 & 91 & 9 \\
\hline $9: 1$ & $84 / \mathrm{F}$ & no & 41 & 43 & 57 \\
\hline Average & - & - & $100^{\mathrm{a}} / 420^{\mathrm{s}}$ & $53^{\mathrm{a}} / 96^{\mathrm{s}}$ & $47^{\mathrm{a}} / 4^{\mathrm{s}}$ \\
\hline & & & & &
\end{tabular}

${ }^{\mathrm{a}}$ asymptomatic sessions, ${ }^{\mathrm{s}}$ symptomatic sessions. 
(a)

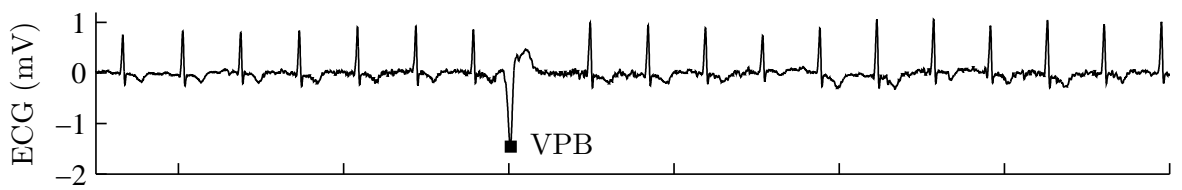

a)
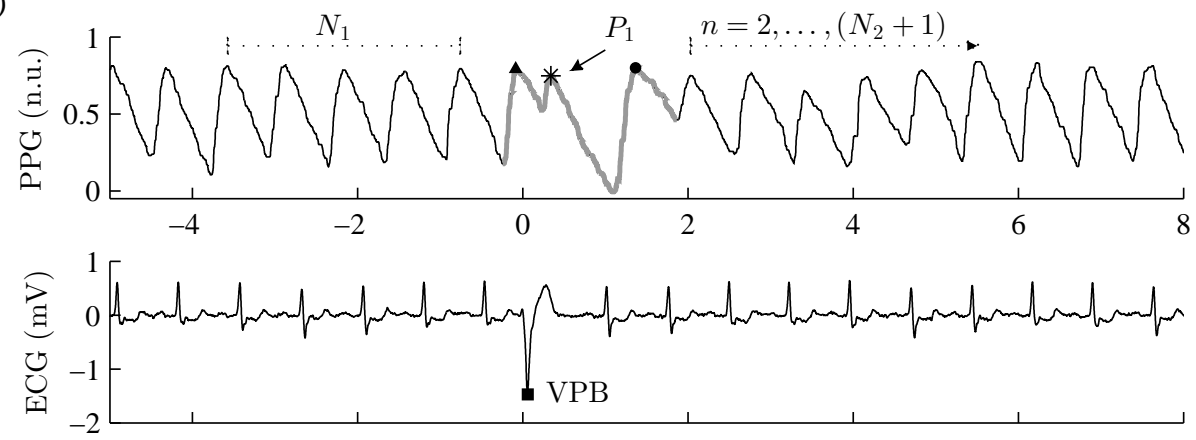

(b)

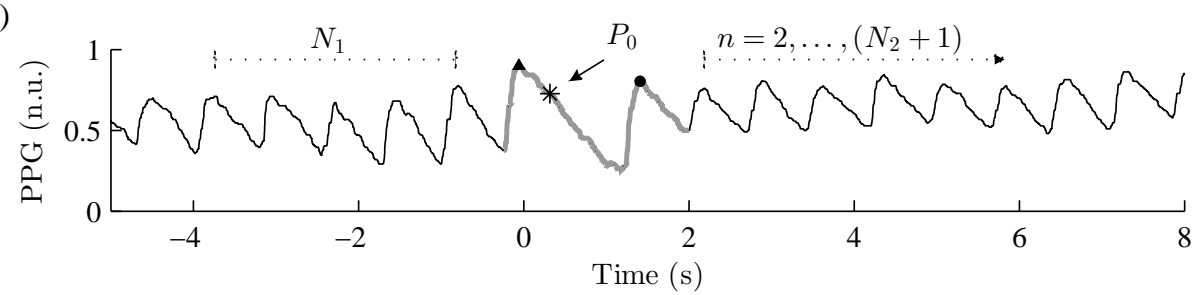

Figure 1: Different PPG pulse patterns caused by a VPB. (a) The $P_{1}$ response contains a low onset-to-peak amplitude pulse $(*)$, partly merged with the normal preceding pulse $(\mathbf{\Delta})$, and (b) $P_{0}$ is a pulseless response, represented by a prolonged compensatory pause that follows a normal PPG pulse $(\boldsymbol{\Delta})$. The first postectopic pulse $(\bullet)$ is not considered in this study.

\subsection{Pulse amplitude recovery}

The characterization of PAR is based on the onset-to-peak upslope amplitude $a_{0}(m)$ of the pulse waveforms following an ectopic beat, but normalized with the amplitude of the pulse waveforms immediately preceding the ectopic beat ( $m$ denotes pulse number). While it is well-known that absolute amplitude measurements of the PPG signal are difficult to interpret, normalization makes it possible to study relative changes in peripheral blood volume. The normalized pulse amplitude, following the $k$-th ectopic beat at time $m=n_{k}$, is defined by

$$
a\left(n_{k}+n\right)=\frac{a_{0}\left(n_{k}+n\right)}{\frac{1}{N_{1}} \sum_{j=1}^{N_{1}} a_{0}\left(n_{k}-j-1\right)}, \quad n=2, \ldots, N_{2}+1,
$$


where $n$ denotes the index of the postectopic beat. The normalizing factor is computed in a window with $N_{1}$ pulses, and the length of the analysis window following the ectopic beat is defined by $\mathrm{N}_{2}$; these parameters were related to the above-mentioned isolation interval used for exclusion of ectopic beats such that $N_{1}=D_{1}-1$ and $N_{2}=D_{2}-1$.

A number of observations should be made regarding the definition in (1). Firstly, the amplitude of the pulse immediately preceding the ectopic beat, i.e., $a_{0}\left(n_{k}-1\right)$, is not used in the normalization factor in order to avoid possible influence by the ectopic beat, e.g., overlapping pulses. Secondly, the amplitude of the ectopic beat, i.e., $a_{0}\left(n_{k}\right)$, irrespective of response type (i.e., $P_{0}$ or $P_{1}$ ), is not used since this beat triggers the drop in blood volume. Thirdly, the amplitude of the beat occurring after the compensatory pause, i.e., $a_{0}\left(n_{k}+1\right)$, is neither used since it has a very high pulse amplitude which, for the present equipment for data acquisition, may be subject to signal saturation.

In order to improve the SNR of the normalized pulse amplitudes, ensemble averaging is performed across all premature ectopic beats, aligned in time with respect to $n_{k}$, i.e.,

$$
\bar{a}(n)=\frac{1}{K} \sum_{k=1}^{K} a\left(n_{k}+n\right), \quad n=2, \ldots, N_{2}+1,
$$

where $K$ denotes the number of premature ectopic beats of the session. The normalized and averaged amplitude $\bar{a}(n)$ is illustrated in Fig. 2 for a session with a considerable drop in relative blood volume-it takes about 7 beats until the amplitude has returned to the level observed before the premature ectopic beats.

Based on $\bar{a}(n)$, two parameters are defined, namely, $\mathcal{A}_{D O}$ reflecting the drop onset in peripheral blood volume caused by a premature ectopic beat,

$$
\mathcal{A}_{D O}=(1-\bar{a}(2)) \cdot 100,
$$

and $\mathcal{A}_{D R}$, reflecting the degree of recovery of reduced peripheral microvascular circulation,

$$
\mathcal{A}_{D R}(W)=\frac{1}{W} \sum_{j=2}^{W+1} \bar{a}(j) \cdot 100,
$$

where $W$ defines the number of postectopic PPG pulses used for averaging. The parameter $\mathcal{A}_{D R}(W)$ ranges from 0 to $100 \%$, where $0 \%$ indicates complete inability to recover to the pre-ectopic amplitude level and 100\% unaltered postectopic peripheral circulation. 


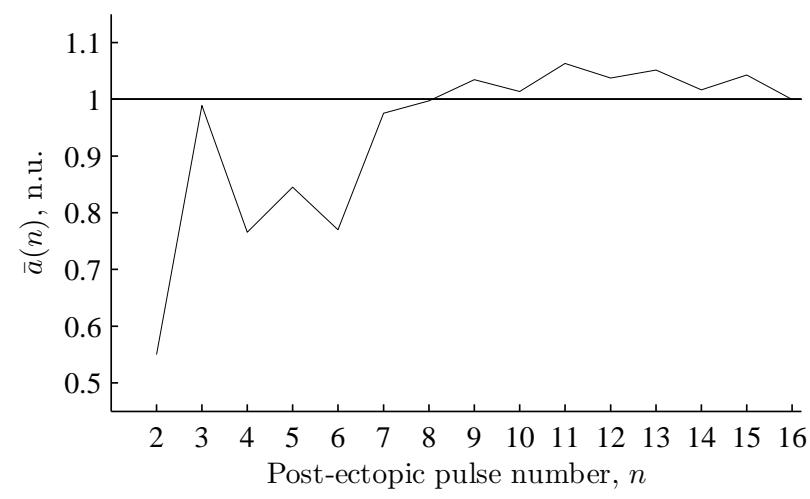

Figure 2: Illustration of the normalized PPG amplitude $\bar{a}(n)$ in a symptomatic session.

Table 2: Statistics of $\mathcal{A}_{D O}$ and $\mathcal{A}_{D R}(W=5)$ based on either VPBs+SVPBs or VPBs only. Statistical differences were determined by the student's $t$-test.

\begin{tabular}{ccccc}
\hline Dataset & PAR $(\%)$ & $\begin{array}{c}\text { Symptomatic } \\
\text { sessions }\end{array}$ & $\begin{array}{c}\text { Asymptomatic } \\
\text { sessions }\end{array}$ & $p$-value \\
\hline VPBs+SVPBs & $\mathcal{A}_{D O}$ & $32 \pm 13$ & $8 \pm 13$ & $<0.005$ \\
& $\mathcal{A}_{D R}$ & $84 \pm 7$ & $93 \pm 4$ & $<0.005$ \\
\multirow{2}{*}{ VPBs } & $\mathcal{A}_{D O}$ & $33 \pm 14$ & $11 \pm 16$ & $<0.05$ \\
& $\mathcal{A}_{D R}$ & $84 \pm 7$ & $91 \pm 4$ & $<0.05$ \\
\hline
\end{tabular}

\section{Results}

The results in Table 2 show that $\mathcal{A}_{D O}$ differs significantly between asymptomatic and symptomatic sessions when computed for all premature ectopic beats. For asymptomatic sessions, the initial drop in peripheral blood volume, as reflected by $\mathcal{A}_{D O}$, is rather small $(8 \pm 13 \%)$, whereas for symptomatic sessions a much larger drop is observed (32 $\pm 13 \%)$. When computing $\mathcal{A}_{D O}$ for VPBs only, the difference between asymptomatic and symptomatic sessions becomes smaller.

When studying the properties of $\mathcal{A}_{D R}(W)$, the number of postectopic PPG pulses $(W)$ needs to first be set. From Fig. 3(a), it is obvious that the postectopic pulse amplitude differs significantly for asymptomatic and symptomatic sessions up until $n=6$, thus suggesting that $W=5$ is a suitable choice in (4), i.e., $\mathcal{A}_{D R} \equiv \mathcal{A}_{D R}(W=5)$ is used in the following. Similar to $\mathcal{A}_{D O}, \mathcal{A}_{D R}$ differs significantly between asymptomatic and symptomatic sessions (Table 2), although 
(a)

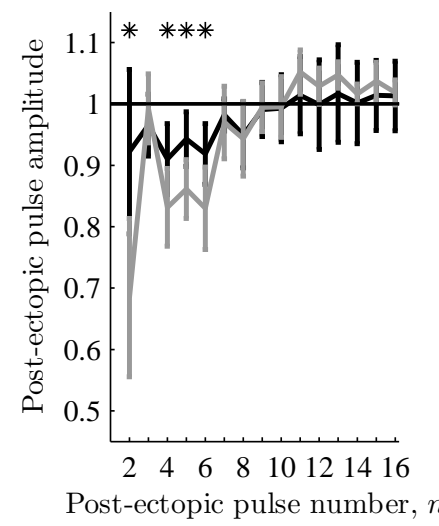

(b)

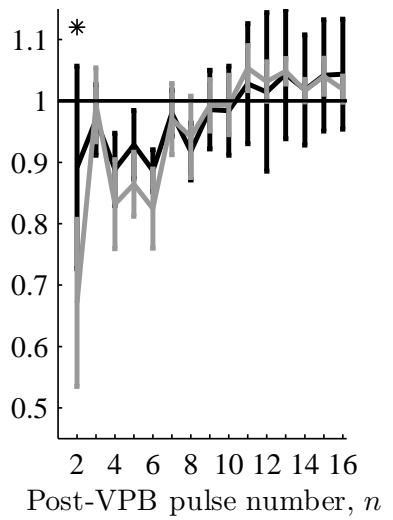

Figure 3: Postectopic pulse amplitude $\bar{a}(n)$ expressed as mean and standard deviation for asymptomatic (black) and symptomatic (grey) treatment sessions when considering (a) VPBs+SVPBs and (b) VPBs. The asterisk indicates statistically significant difference $(p<0.05)$ using the student's $t$-test.

Table 3: Average percentage of the $P_{0}$ and $P_{1}$ responses associated with either VPBs or SVPBs in symptomatic and asymptomatic sessions.

\begin{tabular}{l|ccc|ccc}
\hline \multirow{2}{*}{ Session } & \multicolumn{3}{|c}{ VPBs (\%) } & \multicolumn{3}{c}{ SVPBs (\%) } \\
\cline { 2 - 7 } & $P_{0}$ & $P_{1}$ & indeterm. & $P_{0}$ & $P_{1}$ & indeterm. \\
\hline Sympt. & 82 & 8 & 10 & 58 & 38 & 4 \\
Asympt. & 76 & 16 & 8 & 19 & 60 & 21 \\
\hline
\end{tabular}

the averaged amplitudes $\bar{a}(n), n=4,5,6$, do not differ between asymptomatic and symptomatic sessions when only VPBs are taken into account, see Fig. 3(b).

In the present dataset, the $P_{0}$ response is mostly triggered by VPBs in symptomatic $(82 \%)$ and asymptomatic $(76 \%)$ sessions, and by SVPBs in symptomatic sessions (58\%), see Table 3 . The $P_{1}$ response was found to be the most common in asymptomatic sessions with SVPBs as trigger (60\%).

\section{Discussion}

The study by Zheng et al. [19] appears to be the very first where ectopic beats, as manifested in the PPG signal, are subject to analysis. In that study, the effect of ectopic beat coupling interval on peripheral pulse amplitude was determined, providing useful information for developing a technique to determine the open- 
ing of the aortic valve and the peak filling rate non-invasively and peripherally in patients with frequent ectopic beats. The purpose of the present study is quite different, namely, to introduce a novel method for studying the significance of postectopic pulse amplitude recovery. This method, being defined by a few, simple signal processing blocks, produces two parameters which reflect local changes in the vascular system, with VPBs and SVPBs as triggers. While these parameters are studied here in the context of intradialytic complications during hemodialysis, they may be of interest to investigate in other patient groups where relative changes in peripheral blood volume are expected to play a role.

The results in Table 2 show that symptomatic sessions are associated with a larger $\mathcal{A}_{D O}$ and a smaller $\mathcal{A}_{D R}$ since such sessions contain more VPBs and, therefore, are related to increased hemodynamic instability. When both VPBs and SVPBs are included in the computation of parameters, the postectopic circulation recovery, as reflected by $\bar{a}(n)$, differs significantly between asymptomatic and symptomatic sessions (Fig. 3(a)), while not so when only VPBs are included (Fig. 3(b)). However, it is obvious from Fig. 3(b) that $\bar{a}(n)$ is significantly different only for $n=2$, but not for subsequent pulses. When interpreting this result, it should be recalled that the number of VPBs is lower than the number of VPBs and SVPBs combined.

While the criterion for excluding low SNR segments seems difficult to relax, the isolation interval may be relaxed so that adjacent ectopic beats are allowed to be closer, implying that fewer sessions will have to be excluded. In particular, the present choice of $D_{2}=16$ may be decreased to 10 by accounting for the result in Fig. 3 where $\bar{a}(n)$ reaches steady-state after about 10 pulses. Due to the small size of the dataset, $\mathcal{A}_{D O}$ and $\mathcal{A}_{D R}$ were only investigated in simple statistical terms for assessing intradialytic risk in response to premature ectopic beats, but not for classification/prediction purposes. With the availability of a larger dataset, such purposes deserve to be further investigated.

The method embraces four parameters, namely, $D_{1}, D_{2}, K$, and $W$. Of these parameters, $W$ seems to be the most influential parameter on performance, and, therefore, it was investigated, showing that $W=5$ was a suitable choice. As for the required number of ectopic beats $K$, it is desirable to use a value as large as possible, but a large value also implies that fewer sessions will be eligible for analysis.

It is well-known that hypotension-related symptoms usually occur during the latter part of the treatment session due to the removal of surplus fluids which increases the imbalance in an already impaired cardiovascular system. In the present study, the onset of symptoms was found to often coincide with the onset of an in- 
creasing number of ectopic beats - an observation previously reported in [21] and explored for the purpose of designing a method to predict acute intradialytic hypotension. Hence, in the future, it would be of interest to compare the outcome of using such an approach with the one described in the present paper.

A simple approach would be to the count the number of premature ectopic beats of a session. Interestingly, however, two patients of the present dataset had about the same number of ectopic beats although one session was symptomatic whereas the other was asymptomatic, suggesting that just a simple count may not be useful.

The present study is to investigate whether differences in PAR exist between patients with and without intradialytic complications, using data that derive from the entire treatment session. In a future study, the clinical usefulness of the PAR method should be addressed in further detail, e.g., by computing $\mathcal{A}_{D O}$ and $\mathcal{A}_{D R}$ from data acquired during the first $30 \mathrm{~min}$ of the session when complications are unlikely to occur. The resulting parameter values can then be employed for predicting complications during the remaining session so that suitable preventive measures can be taken at an early stage.

The PPG signal was sampled at a rate of $1000 \mathrm{~Hz}$, i.e., much higher than needed. The proposed method is not critically dependent on a high temporal precision of the PPG signal when it comes to PAR analysis. Thus, the use of a lower PPG sampling rate, e.g., $250 \mathrm{~Hz}$, is expected to produce similar results. It should be emphasized that the ECG was used for annotation of the ectopic beats in the PPG signal. In a clinical context, ectopic beats can be detected directly from the PPG signal [11, 22], making the ECG signal unnecessary.

\section{Limitations}

The present results are preliminary in nature, deriving from a small dataset, and serves to illustrate the use of a new method. A much larger dataset will have to be acquired, with both hypotension-prone and -resistant patients, so that the significance of the method can be fully evaluated. With the advent of a larger dataset, the analysis of only one session per patient should be implemented. Finally, a larger dataset would be helpful in providing information on how to select the parameters of the method.

\section{Conclusions}

A novel approach to characterizing postectopic recovery in the PPG signal is introduced and illustrated. The results suggest that asymptomatic and symp- 
tomatic sessions of patients undergoing hemodialysis treatment are associated with different normalized pulse amplitude patterns. The significance of postectopic pulse amplitude recovery may be explored in other applications where relative changes in microvascular peripheral blood volume are expected to play a role.

\section{Acknowledgements}

The authors are grateful to Dr. K. Solem, Baxter-Gambro, Lund, Sweden, for valuable comments on the manuscript. The authors also thank Dr. S. Ladefoged and Dr. J. Cordtz at Rigshospitalet, Copenhagen, for being supportive of the data acquisition.

This work was supported by the Swedish Institute (00923/2011), Ministerio de Economa y Competitividad (MINECO), and FEDER (TIN2014-53567-R).

\section{References}

[1] C. McIntyre, "Effects of hemodialysis on cardiac function," Kidney Int., vol. 76, no. 4, pp. 371-375, 2009.

[2] D. Green, P. R. Roberts, D. I. New, and P. A. Kalra, "Sudden cardiac death in hemodialysis patients: An in-depth review," Am. J. Kidney Dis., vol. 57, no. 6, pp. 921-929, 2011.

[3] M. Shamseddin and P. Parfrey, "Sudden cardiac death in chronic kidney disease: epidemiology and prevention," Nat. Rev. Nephrol., vol. 7, no. 3, pp. 2712-22, 2011.

[4] O. M. Shapira and Y. Bar-Khaym, "ECG changes and cardiac arrhythmias in chronic renal failure patients on hemodialysis," J. Electrocardiol., vol. 25, no. 4, pp. 273-279, 1992.

[5] S. Abe, M. Yoshizawa, N. Nakanishi, T. Yazawa, K. Yokota, M. Honda, and G. Sloman, "Electrocardiographic abnormalities in patients receiving hemodialysis," Am. Heart J., vol. 131, no. 6, pp. 1137-1144, 1996.

[6] A. Santoro, E. Mancini, R. Gaggi, S. Cavalanti, S. Severi, R. Cagnoli, F. Badiali, B. Perrone, G. London, H. Fessy, L. Mercadal, and F. Grandi, "Electrophysiological response to dialysis: the role of dialysate potassium content and profilling," Contrib. Nephrol., vol. 149, pp. 295-305, 2005. 
[7] G. Schmidt, M. Malik, P. Barthel, R. Schneider, K. Ulm, L. Rolnitzky, A. J. Camm, J. T. Bigger Jr, and A. Schömig, "Heart-rate turbulence after ventricular premature beats as a predictor of mortality after acute myocardial infarction," Lancet, vol. 353, pp. 1390-1396, 1999.

[8] K. Solem, P. Laguna, J. P. Martínez, and L. Sörnmo, "Model-based detection of heart rate turbulence," IEEE Trans. Biomed. Eng., vol. 55, no. 12, pp. 2712-2722, 2008.

[9] F. Sandberg, R. Bailón, D. Hernando, P. Laguna, J. P. Martínez, K. Solem, and L. Sörnmo, "Prediction of hypotension in hemodialysis patients," Phys. Meas., vol. 35, no. 9, pp. 1885-1898, 2014.

[10] A. Celik, M. Melek, S. Yuksel, E. Onrat, and A. Avsar, "Cardiac autonomic dysfunction in hemodialysis patients: The value of heart rate turbulence," Hemodial. Int., vol. 15, no. 2, pp. 193-199, 2011.

[11] E. Gil, P. Laguna, J. P. Martínez, Ó. Barquero-Pérez, A. García-Alberola, and L. Sörnmo, "Heart rate turbulence analysis based on photoplethysmography," IEEE Trans. Biomed. Eng., vol. 60, no. 11, pp. 3149-3155, 2013.

[12] J. Allen, "Photoplethysmography and its application in clinical physiological measurement," Physiol. Meas., vol. 28, no. 3, pp. R1-39, 2007.

[13] C. Ahlström, A. Johansson, F. Uhlin, T. Länne, and P. Ask, "Noninvasive investigation of blood pressure changes using the pulse wave transit time: a novel approach in the monitoring of hemodialysis patients," J. Artif. Organs, vol. 8, no. 3, pp. 192-197, 2005.

[14] F. Javed, P. M. Middleton, P. Malouf, G. S. H. Chan, A. V. Savkin, N. H. Lovell, E. Steel, and J. Mackie, "Frequency spectrum analysis of finger photoplethysmographic waveform variability during hemodialysis," Physiol. Meas., vol. 31, no. 9, pp. 1203-1216, 2010.

[15] A. Burkert, A. Scholtze, and M. Tepel, "Noninvasive continuous monitoring of digital pulse waves during hemodialysis," ASAIO J., vol. 52, no. 2, pp. 174-179, 2006.

[16] F. Sandberg, M. Holmer, B. Olde, and K. Solem, "Estimation of respiratory information from the built-in pressure sensors of the dialysis machine," in Proc. Comput. Cardiol., vol. 41, pp. 853-856, 2014. 
[17] B. Fuehrlein, R. Melker, and E. Ross, "Alar photoplethysmography: A new methodology for monitoring fluid removal and carotid circulation during hemodialysis," J. Clin. Monit. Comput., vol. 21, no. 4, pp. 211-228, 2007.

[18] K. Solem, B. Olde, and L. Sörnmo, "Prediction of intradialytic hypotension using photoplethysmography," IEEE Trans. Biomed. Eng., vol. 57, no. 7, pp. 1611-1619, 2010.

[19] D. Zheng, J. Allen, and A. Murray, "Determination of aortic valve opening time and left ventricular peak filling rate from the peripheral pulse amplitude in patients with ectopic beats," Physiol. Meas., vol. 29, no. 12, pp. 14111419, 2008.

[20] J. Lázaro, E. Gil, J. M. Vergara, and P. Laguna, "Pulse rate variability analysis for discrimination of sleep-apnea-related decreases in the amplitude fluctuations of pulse photoplethysmographic signal in children," IEEE $J$. Biomed. Health Inform., vol. 18, no. 1, pp. 240-246, 2014.

[21] K. Solem, A. Nilsson, and L. Sörnmo, "An ECG-based method for early detection of abrupt changes in blood pressure during hemodialysis," ASAIO J., vol. 52, no. 3, pp. 282-290, 2006.

[22] A. Solosenko, A. Petrėnas, and V. Marozas, "Photoplethysmography-based method for automatic detection of premature ventricular contractions," IEEE Trans. Biomed. Circ. Syst., vol. 9, pp. 662-669, 2015. 